\title{
Usefulness and Reliability of Vectorcardiography in the Diagnosis of Myocardial Infarction
}

\section{A Vectorcardiographic and Pathological Correlation}

\author{
Kazuhiko Murata, M.D., Hiroshi Kurihara, M.D., \\ Satoru Matsushita, M.D., Fujio Terasawa, M.D., \\ Masao Ikeda, M.D. and Masuji SEKr, M.D.
}

In order to determine the sensitivity and reliability of vectorcardiography in the diagnosis of myocardial infarction, Frank lead vectorcardiograms of autopsy cases were reviewed and the results were compared with the pathologic findings. The diagnosis of myocardial infarction was possible in 30 of 43 autopsy-proved cases of myocardial infarction. Two of these 30 cases were not diagnosed by routine electrocardiogram. On the other hand, a false diagnosis of myocardial infarction was made by vectorcardiogram in 11 of 305 cases without extensive myocardial fibrosis at later autopsy. Although vectorcardiography is a sensitive diagnostic method in myocardial infarction, a possibility of overdiagnosis should not be neglected.

$\mathrm{T}$

HE purpose of the present paper is to determine the diagnostic sensitivity and accuracy of vectorcardiography in myocardial infarction. Although there are several reports which indicate the superior sensitivity of vectorcardiography to routine electrocardiography in the diagnosis of myocardial infarction, only a few studies were made on autopsy materials. ${ }^{1 /-3}$ ) Moreover, reports on the possibility of vectorcardiographic overdiagnosis are still sparse. ${ }^{4-7)}$ In the present study, the vectorcardiograms and electrocardiograms of the autopsy cases were reviewed and the results were compared with the pathologic findings.

\section{Materials and Methods}

The materials consisted of 348 autopsy cases in which a Frank lead vectorcardiogram had been recorded. The vectorcardiograms and routine 12-lead electrocardiograms of these cases were reviewed independently and the cases with the findings interpreted as indicative of myocardial infarction were selected. The autopsy records were checked thereafter.

The method of recording the vectorcardiogram has been described before. ${ }^{8)}$

From the Third Department of Internal Medicine, Faculty of Medicine, University of Tokyo and the Yokufukai Geriatric Hospital, Tokyo.

Received for publication December 6, 1966. 
Although the interpretation of the vectorcardiogram was mainly based on the criteria described by the previous investigators, ${ }^{1,2), 9\}-11)}$ three vectorcardiograms were interpreted as indicative of myocardial infarction on the basis of a large indentation at the efferent limb of the QRS loop.

At autopsy, the heart was examined according to our routine method. The coronary arteries were sectioned transversely at intervals of 0.3 to $0.5 \mathrm{~cm}$., while the myocardium was sectioned at intervals of $0.5 \mathrm{~cm}$. A significant coronary sclerosis was designated when the reduction of the diameter of the lumen of the coronary artery was 50 per cent or more. A myocardial infarction, defined as an extensive myocardial fibrosis extending at least $2 \mathrm{~cm}$. in one dimension, was demonstrated in 43 of 348 cases.

\section{Results}

1. Diagnostic sensitivity

The routine electrocardiogram showed a diagnostic change in 28 of 43 cases of proved myocardial infarction as seen in Table $\mathrm{I}$.

Table I. Diagnostic Sensitivity of Vectorcardiography and Electrocardiography in Myocardial Infarction

\begin{tabular}{|c|c|c|c|c|}
\hline & \multirow{2}{*}{$\begin{array}{c}\text { Number of } \\
\text { cases }\end{array}$} & \multicolumn{3}{|c|}{ Diagnosed by } \\
\hline & & $\begin{array}{l}\text { ECG } \\
\text { only }\end{array}$ & $\begin{array}{l}\text { VGG } \\
\text { only }\end{array}$ & $\mathrm{ECG} \& \mathrm{VGG}$ \\
\hline $\begin{array}{l}\text { Anterior } \\
\quad \text { (incl. Anteroseptal and Antero- } \\
\text { lateral) }\end{array}$ & 19 & 0 & 0 & 15 \\
\hline $\begin{array}{l}\text { Inferior or Posterior } \\
\text { (incl. Inferolateral and Postero- } \\
\text { lateral) }\end{array}$ & 19 & 0 & 1 & 12 \\
\hline Lateral & 3 & 0 & 0 & 1 \\
\hline Extensive, subendocardial & 2 & 0 & 1 & 0 \\
\hline Total & 43 & 0 & 2 & $\frac{28(65.1 \%)}{(69.8 \%)}$ \\
\hline
\end{tabular}

The Frank lead vectorcardiograms of all these 28 cases were also interpreted as indicative of myocardial infarction. On the other hand, there were 2 cases in which a diagnosis of myocardial infarction was suspected only in the vectorcardiogram while the routine electrocardiogram was not contributory. In these 2 cases, myocardial infarction was seriously suspected on the basis of the abnormal configuration of the QRS loop, although the direction of the initial vector was within normal limits. No significant $Q$ wave was observed in the routine electrocardiogram in both cases. The vectorcardiogram and electrocardiograms of these cases are illustrated in Figs. 1 and 2. 


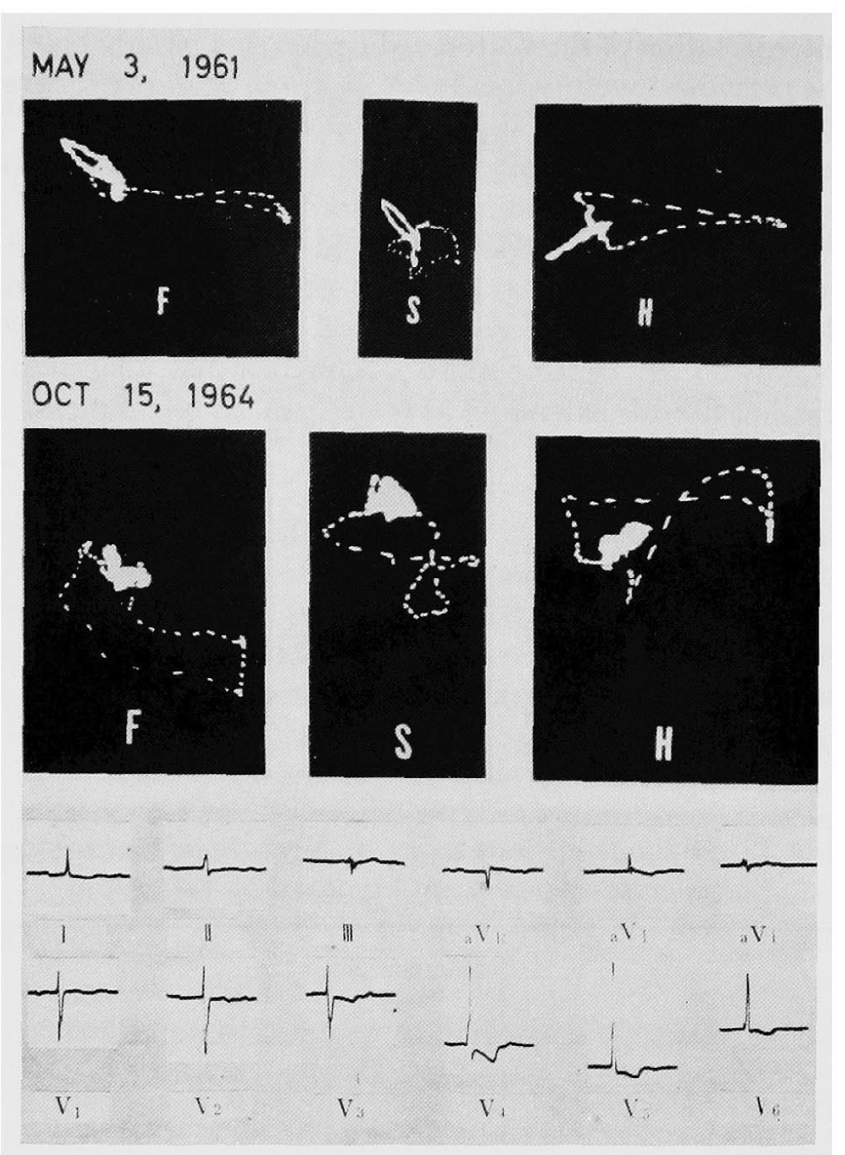

Fig. 1. Vectorcardiograms and electrocardiogram of a woman died at the age of 79. The upper vectorcardiogram recorded in 1961 merely showed a pattern of left ventricular hypertrophy. In the lower tracing recorded 3 years later, an unusual deformity of the QRS loop was observed. There was a large indentation at the efferent limb of the loop. Although no clear-cut evidence of myocardial infarction was recognized in the routine electrocardiogram recorded at that time, the presence of lateral infarction was seriously considered on the basis of the abnormal vectorcardiographic finding. Old myocardial infarction extending from the lateral to the posterior wall of the left ventricle was demonstrated at post-mortem study.

\section{False positivity}

The vectorcardiograms of 11 of 305 cases without extensive myocardial fibrosis at later autopsy was erroneously believed to be diagnostic of myocardial infarction. Figs. 3 and 4 are the illustrative vectorcardiograms.

Nine of these 11 cases were misdiagnosed as old anteroseptal or anterolateral infarction on the basis of abnormal orientation of the initial QRS vector and the unusual configuration of the initial part of the QRS loop. The vectorcardiograms of the remaining 2 cases were considered to be diagnostic of 

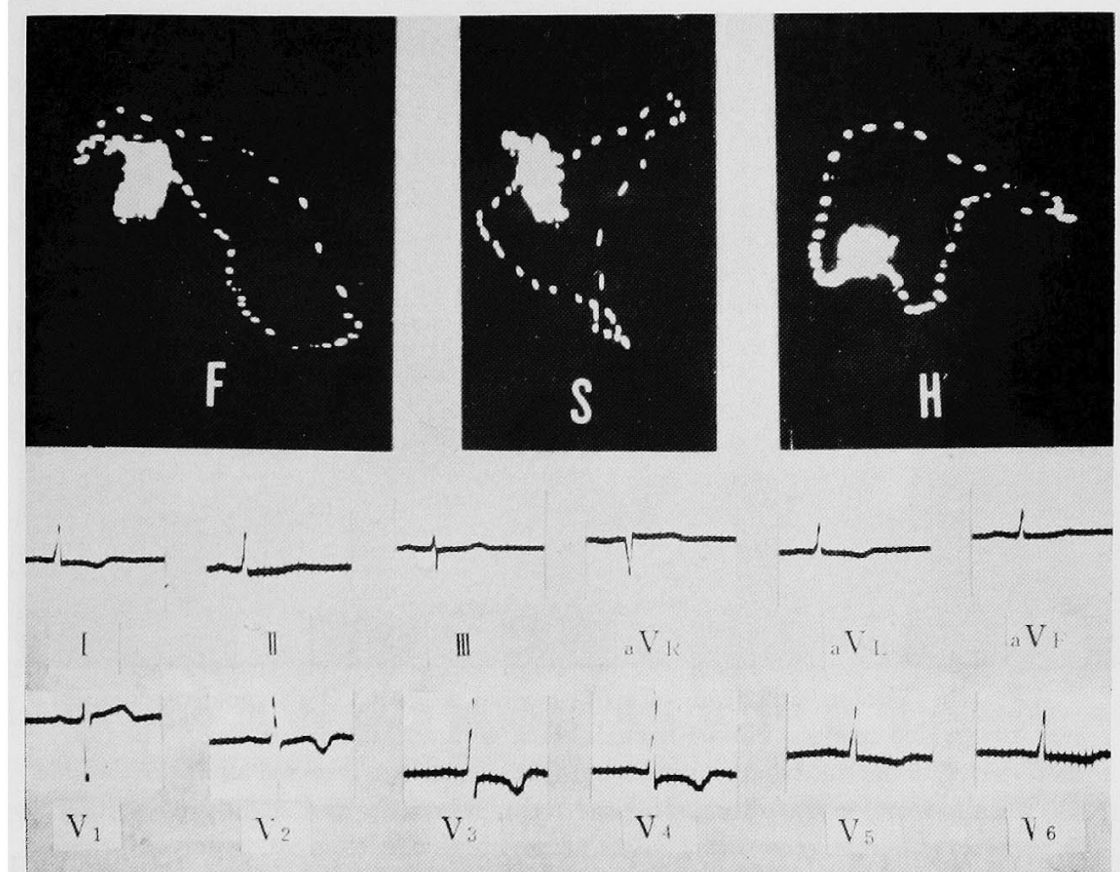

III

. V

a
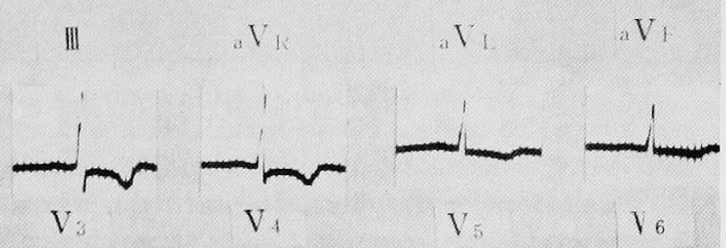

Fig. 2. Vectorcardiogram of a 85-year-old woman. Although the 12-lead electrocardiogram was not diagnostic, old myocardial infarction, probably involving the lateral wall, was suspected in this case on the basis of the unusually distorted QRS loop. Old extensive subendocardial infarction was demonstrated at autopsy.

inferior infarction because the initial portion of the QRS loop was displaced superiorly.

At autopsy, there was an extensive infiltration of amyloid in myocardium in 1 case, but no extensive myocardial lesion corresponding to the vectorcardiographic finding was demonstrated in the others. Scattered fibrosis of myocardium was present in 4 . Coronary sclerosis of significant degree was observed in 8 and a mild to moderate left ventricular hypertrophy was present in 5. Pulmonary emphysema of moderate to severe degree was observed in 8 cases. A giant right atrium, significant right ventricular hypertrophy or marked hypertrophy of the interventricular septum was not demonstrated.

\section{Discussion}

The sensitivity of electrocardiography or vectorcardiography in the diagnosis of myocardial infarction has been known to be between 60 and 80 per cent, ${ }^{1,3), 12)-16)}$ and the present results are in good agreement with the previous reports. The correct diagnosis of myocardial infraction was made 


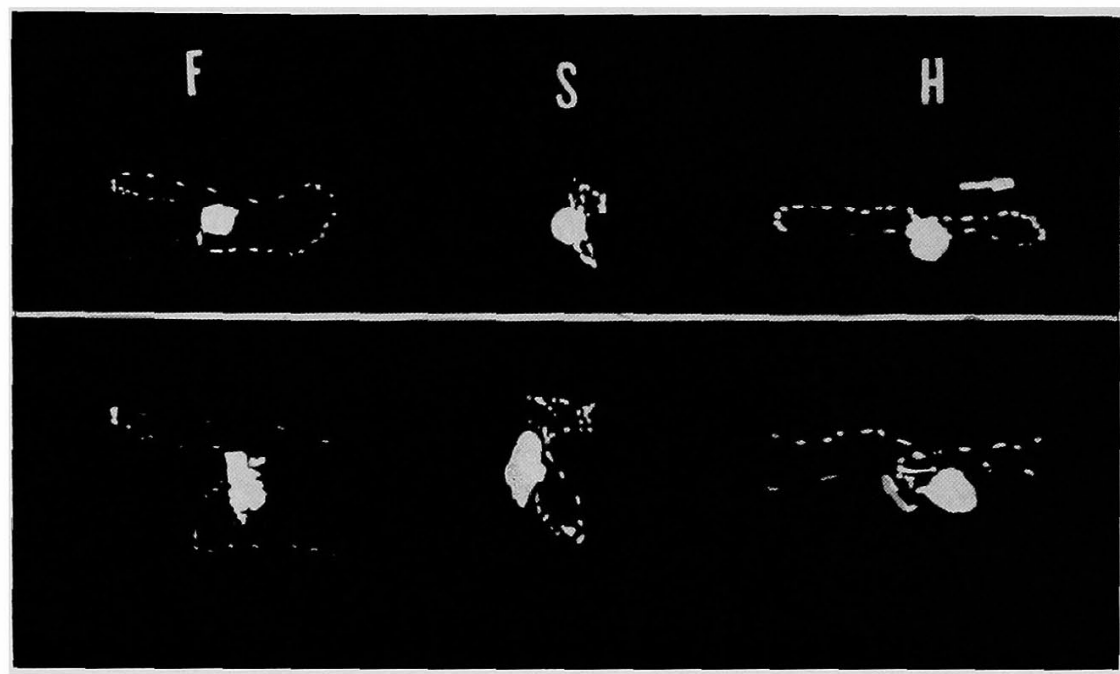

FIG. 3. Vectorcardiogram of a 87-year-old woman. This vectorcardiogram was interpreted as right bundle branch block with anterior infarction. The QRS loop started to the right and no significant $Q R S$ vector was directed anteriorly, while the terminal vector was directed to the right, superiorly and slightly posteriorly. A vectorcardiogram recorded 2 years before showed a classical pattern of right bundle branch block. At autopsy, no significant myocardial lesion was demonstrated, although moderate coronary sclerosis and severe pulmonary emphysema were observed.
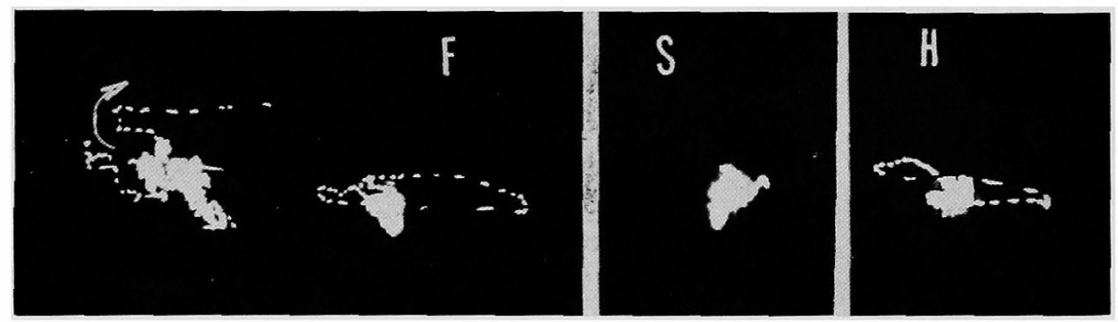

Fig. 4. Vectorcardiogram of a 82-year-old man. This tracing was interpreted as old inferior infarction. There was a large initial vector directed superiorly. Significant Q waves were observed in leads II, III and aVf. There was no significant myocardial lesion at autopsy.

in the vectorcardiogram in 69.8 per cent of 43 cases with autopsy proved myocardial infarction.

In the present series, there were 2 cases in which a diagnosis of myocardial infarction was suspected only by the vectorcardiogram while the routine electrocardiogram was not contributory. The diagnosis of myocardial infarction in these cases was based on the abnormal configuration of the initial portion of the QRS loop, although the direction of the initial QRS vector was within normal range. This sort of abnormality is usually not recognized in the routine electrocardiogram. 
Although the above observation suggests the superior sensitivity of vectorcardiography to routine electrocardiography in the diagnosis of myocardial infarction, a possibility of overdiagnosis based solely on this kind of unusual configuration of the QRS loop remains to be investigated. A marked distortion of the QRS loop of such a degree as shown in this paper has not been seen in the absence of myocardial infarction. However, a small indentation in the initial or middle portion of the QRS loop was observed not infrequently in the cases without myocardial lesion at later autopsy. Similar observations were reported by Ueda and associates. ${ }^{17}$

On the other hand, the presence of 11 cases with false positive diagnosis in vectorcardiogram in the present series may be discouraging. A possibility of this sort of overdiagnosis has not been studied systematically up to the present time, although Abramson, " Banta and Estes, ${ }^{5)}$ Hugenholtz and associates, ${ }^{2)}$ and Toyama" ${ }^{7}$ reported that a vectorcardiogram may mimic myocardial infarction in the cases of giant right atrium or hypertrophy of the interventricular septum, or, rarely, even in apparently normal persons. Recently, Simonson and associates ${ }^{18)}$ reported that a myocardial infarction was not infrequently diagnosed erroneously in cases of left ventricular hypertrophy.

It is not at all surprising to find a vectorcardiographic pattern simulating myocardial infarction in the presence of extensive infiltration of amyloid in the myocardium, the mechanism of the development of the unusual vectorcardiographic findings in the remaining 10 cases in our present series is not entirely clear. A giant right atrium or significant septal hypertrophy was not observed in the present series. From a high incidence of coronary sclerosis and pulmonary emphysema, an altered position of the heart or an unusual intraventricular conduction may be postulated to be major contributing factors. Although the underlying mechanism remains obscure, in reading vectorcardiograms a possibility of overdiagnosis of myocardial infarction should be borne in mind.

\section{SUMMARY}

(1) Frank lead vectorcardiograms of 348 autopsy cases, including 43 cases with myocardial infarction, were reviewed and the results were compared with the pathologic observations.

(2) The correct diagnosis of myocardial infarction was possible in 30 of 43 autopsy-proved cases in the vectorcardiogram. Two of these 30 cases were not diagnosed by the routine electrocardiogram. On the other hand, the vectorcardiogram missed a diagnosis of myocardial infarction in none of the 28 cases of autopsy-proved myocardial infarction diagnosed by routine electro- 
cardiography.

(3) The overdiagnosis of myocardial infarction in the vectorcardiography was made in 11 of 305 cases without extensive myocardial fibrosis at later autopsy. Nine of these 11 cases were misdiagnosed as anterior infarction, while the remaining 2 were misinterpreted as inferior infarction.

(4) The necessity of considering the possibility of overdiagnosis of myocardial infarction in reading vectorcardiograms was pointed out, although the present observations suggest the superior sensitivity of vectorcardiography to routine electrocardiography.

\section{ACKnowledgement}

The authors thank Prof. Kiku Nakao and Dr. Fujiro Amako for critically reviewing the manuscript.

\section{REFERENCES}

1. Hugenholtz, P. G., Whipple, G. H., and Levine, H. D.: Circulation 24 : 808, 1961.

2. Hugenholtz, P. G., Forkner, C. E., Jr., and Levine, H. D.: Girculation 24 : 823, 1961.

3. Wolff, L. et al. : Circulation $23: 861,1961$.

4. Abramson, H. : Canad. Med. Assoc. J. $90: 903,1964$.

5. Banta, H. D. and Estes, E. H., Jr.: Am. J. Cardiol. 14: 218, 1964.

6. Surawicz, X., Van Horne, R. G., Urbach, J. R., and Bellet, S. : Circulation 12: 391, 1955.

7. Toyama, S. : Jap. Circulat. J. $25: 155,1961$.

8. Murata, K., Kurihara, H., Hosoda, S., Ikeda, M., and Seki, M. : Jap. Heart J. 5 : 543, 1964.

9. Hoffman, I., Taymor, R. C., Morris, M. H., and Kittel, I. : Am. Heart J. 79: 295, 1965.

10. Hoffman, I., Taymor, R. C., and Cootnick, A.: Circulation 29: 562, 1964.

11. Hugenholtz, P. G., Ryan, T.J., Woerner, T., and Levine, H. D.: Circulation $27: 386,1963$.

12. Burch, G. E., Horan, L. G., and Ziskind, J. : Girculation 18 : 325, 1958.

13. Kurihara, H., Kuramoto, K., Murata, K., Terasawa, F., Seki, M., and Ikeda, M.: Jap. Heart J. $8: 419,1967$.

14. Lee, K. T., Thomas, W. A., Rabin, E. R., and O'Neal, R. M.: Circulation 15: 197, 1957.

15. Niitani, H. et al. : Jap. J. Clin. Med. 18: 1013, 1960 (in Japanese).

16. Weiss, M. M. and Weiss, M. M., Jr. : Arch. Int. Med. 101 : 1126, 1958.

17. Ueda, H., Mashima, S., and Yanai, Y.: Jap. J. Clin. Med. 22 : 2464, 1964 (in Japanese).

18. Simonson, E., Tuna, N., Okamoto, N., and Toshima, H.: Am. J. Cardiol. $17:$ 829, 1966. 